\title{
DISCURSO INAUGURAL CONMEMORACIÓN DEL XXX ANIVERSARIO CENTRO DE INVESTIGACIÓN EN NUTRICIÓN ANIMAL ${ }^{1}$
}

M.Sc. Augusto Rojas-Bourillón²

Es un honor hoy, representar a un grupo de profesores multidisciplinario de la Escuela de Zootecnia de la Universidad de Costa Rica; quienes deciden transformar un simple laboratorio de análisis de plantas forrajeras, en lo que hoy conocemos como el Centro de Investigación Animal (CINA), atendiendo la transformación que se necesitaba para el país en su competitividad en lo pertinente al control de calidad en alimentos para animales.

Todos los días se demuestra con acciones, los aportes del CINA en la calidad e inocuidad de los mismos. Los datos analíticos de alta calidad y confiabilidad acerca de productos de consumo animal que provee el CINA como parte de un sistema de control continuo, aporta información muy valiosa para socavar distintas dudas que se les genera a los consumidores y a las compañías que se dedican a la fabricación de estos productos.

Mediante esta información y como parte del valioso apoyo que se le da a las autoridades estatales, como país somos capaces de tomar medidas preventivas y mantener altos niveles de calidad en los productos que son consumidos por animales domésticos.

La existencia de un sistema establecido y funcional es crucial, para eliminar y evitar la contaminación de alimentos que puedan representar un peligro para la salud animal, de esta forma, podemos sintetizar nuestro aporte en garantizar y proteger la salud pública por medio de la nutrición animal.

Durante estos treinta años, el CINA ha venido consolidando en conjunto con la Escuela de Zootecnia líneas de investigación en las que ha permitido generar ciencia que aporta al desarrollo del país, mediante los resultados de dichas investigaciones y con la inserción de profesionales entrenados en nuevas técnicas de producción enfocadas a la resolución de problemas propios de la actividad pecuaria.

\footnotetext{
${ }^{1}$ Discurso pronunciado el 1 de octubre de 2015, en el Auditorio de la Ciudad de la Investigación. Universidad de Costa Rica.

${ }^{2}$ Universidad de Costa Rica, Centro de Investigación en Nutrición Animal y Escuela de Zootecnia. Para correspondencia: augusto.rojas@ucr.ac.cr

Recibido: 1 de octubre 2015

Aceptado: 1 octubre 2015
} 
Énfasis en la comprensión nutricional de los forrajes, alternativas para los desechos agropecuarios, prevención de desordenes metabólicos, manejo apropiado del agua, estrategias nutricionales para mitigar la emisión de metano, entre otros, son algunos de los ejemplos que se pueden mencionar.

En este sentido, el CINA ha liderado y sigue liderando todo lo relacionado con la nutrición animal, la inocuidad química y microbiológica de alimentos para animales, no sólo a nivel nacional, sino a nivel regional.

El CINA también ha procurado la transferencia efectiva de la información; no solo se debe transparencia y apertura con respecto a la información generada para las entidades oficiales, sino además el quehacer del CINA es de transferir el conocimiento generado y sus recursos, a través de publicaciones científicas y entrenamientos a los productores nacionales o a las plantas de alimentos, sino también a nivel internacionales.

Actualmente tenemos alrededor de 56 proyectos vigentes, en algunos de los cuales estamos vinculados con algunos entes internacionales y la Universidad Nacional y el Instituto Tecnológico de Costa Rica. No sólo es importante mencionar los logros, sino más bien, el enfoque a futuro para hacer frente a los requerimientos y valores comerciales que cada día son más en materia de la producción animal.

En ese aspecto, contar la presencia de personal altamente capacitado y la opción y validación de técnicas de laboratorio que permitan generar más conocimiento tecnológico al servicio del sector productivo industrial, así como mejorar el entrenamiento de los profesionales en zootecnia como responsables de la producción pecuaria, son metas que el CINA se ha establecido como parte de los aportes establecidos de la Universidad de Costa Rica para el país.

Esto se ha venido logrando con el valioso aporte de la Vicerrectoría de Investigación, la Comisión de Equipamiento y la Rectoría quienes desean que el CINA se posicione cada vez más como referente a nivel nacional en materia de nutrición animal. 
Si bien, esta actividad genera satisfacción, debemos mencionar que en días pasados, las atenciones se dieron debido al fallecimiento del M.Sc. Jorge Sánchez González quien fue director del CINA y de la Escuela de Zootecnia, entre sus logros importantes, además de sus aportes en temas de nutrición mineral y desordenes metabólicos, podemos mencionar la firma de un convenio entre la Universidad y el Ministerio de Agricultura y Ganadería.

Por último, agradecerles por su presencia el día hoy. Hay que recalcar el concepto de puertas abiertas que el CINA ha mantenido durante todos estos años y seguirá manteniendo, también nuestra anuencia de colaboración para resolver problemas nacional en conjunto con otros centros de investigación para impulsar el desarrollo del país. ¡Muchas Gracias! 